\begin{tabular}{|c|c|c|c|c|}
\hline & $\begin{array}{l}\text { ELSEVIER } \\
\text { JOURNAL CO }\end{array}$ & $\begin{array}{l}\text { SCIENCE B.V. [DTD } 4.1 .0] \\
\text { MPNW ARTICLE No. } 2466\end{array}$ & COMPNW 2460 & \\
\hline & PAGES 01-11 & DISPATCH 7 February 2001 & PROD. TYPE: FROM Disk & \\
\hline
\end{tabular}

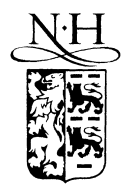

ELSEVIER

\title{
Provision of quality of service for active services
}

\section{Abstract}

A novel approach to quality of service control in an active service network (application layer active network) is 7 described. The approach makes use of a distributed genetic algorithm based on the unique methods that bacteria use to 8 transfer and share genetic material. We have used this algorithm in the design of a robust adaptive control system for

9 the active nodes in an active service network. The system has been simulated and results show that it can offer clear differentiation of active services. The algorithm places the right software, at the right place, in the right proportions;

11 allows different time dependencies to be satisfied and simple payment related increases in performance. (C) 2001 Elsevier

12 Science B.V. All rights reserved.

13 Keywords: Network management; Genetic algorithms; ALAN

\section{1. Introduction}

To be popular with customers an active service platform must provide some clear service quality assurances. Users of an active service network supply the programs and policies required for their custom services in transport packets alongside their data. Clearly it should be possible for these users to specify the Quality of Service (QoS) using any metric that is important to them. The rate of loss of packets carrying service requests or policies, and the service response time (latency) are two obvious examples. In this paper we discuss the management of QoS in an application layer active network (ALAN) [1] that enables users to place software (application layer services) on servers embedded in the network. Despite the obvious virtual networking overheads, the resulting end to

\footnotetext{
${ }^{*}$ Corresponding author.

E-mail addresses: ian.w.marshall@bt.com (I. W Marshall), christopher.roadknight@bt.com (C. Roadknight).
}

end service performance will often be significantly 31 better than if the services executed in the user's end 32 systems (as at present). For example, a network 33 based conference gateway can be located so as to 34 minimise the latency of the paths used in the 35 conference, whereas an end system based gateway 36 will usually be in a sub-optimal location.

For the purposes of this work we have assumed that the latency and loss associated with the network based servers is significantly greater than the latency and loss associated with the underlying network. In the case of latency this is clear packet handling times in broadband routers are around $10 \mu \mathrm{s}$, whilst the time taken to move a packet into the user space for application layer processing is a few milliseconds. In the case of loss the situation is less clear since currently servers do not drop requests, they simply time-out. However, measurement of DNS lookup [2] suggest DNS time-outs due to server overloads occur significantly more frequently than DNS packet losses, so we feel our assumption is reasonable. 
In Section 2 we briefly describe our active services platform ALAN and its associated management system. We then justify our approach to QoS in an ALAN environment. We then describe a novel control algorithm, which can control QoS in the desired manner. Finally we show the results of some simulations using the novel algorithm. The results are very encouraging and illustrate for the first time that a distributed AI approach may be a productive QoS management tool in an active services network. However, further work is required before we can justify the use of our approach in a working active network.

\section{ALAN}

ALAN [1] is based on users supplying java based active code (proxylets) that runs on edge systems (dynamic proxy server - DPS) provided by network operators. Messaging uses HTML/XML and is normally carried over HTTP. There are likely to be many DPSs at a physical network node (at least one for each service provider using the node). It is not the intention that the DPS is able to act as an active router. ALAN is primarily an active service architecture, and the discussion in this paper refers to the management of active programming of intermediate servers. Fig. 1 shows a schematic of a possible DPS management architecture.

The DPS has an autonomous control system that performs management functions delegated to it via policies (scripts and pointers embedded in

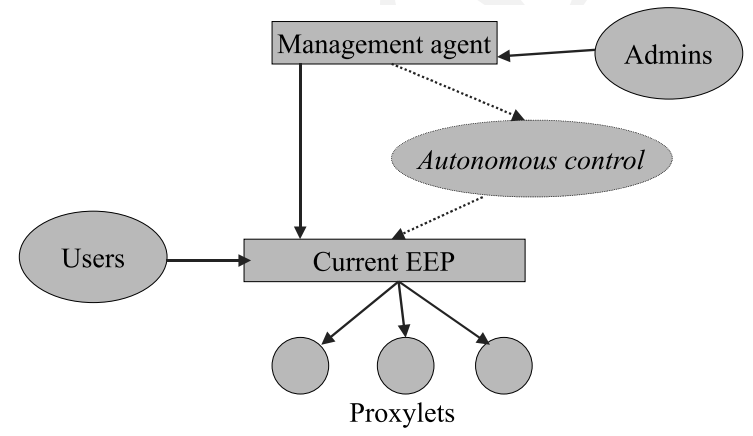

Fig. 1. Schematic of proposed ALAN design.
XML containers). Currently the control system 84 supports a conventional management agent inter- 85 face that can respond to high level instructions 86 from system operators [3]. This interface is also 87 open to use by users (who can use it to run pro- 88 grams/active services) by adding a policy pointing 89 to the location of their program and providing an 90 invocation trigger. Typically the management 91 policies for the program are included in an XML 92 metafile associated with the code using an XML 93 container [4,5], but users can also separately add 94 management policies associated with their pro- 95 grams using HTTP post commands. In addition 96 the agent can accept policies from other agents and 97 export policies to other agents. This autonomous 98 control system is intended to be adaptive. 99

Not shown in the figure are some low level 100 controls required to enforce sharing of resources 101 between users, and minimise unwanted interac- 102 tions between users. There is a set of kernel level 103 routines [6] that enforce hard scheduling of the 104 system resources used by a DPS and the associated 105 virtual machine that supports user supplied code. 106 In addition the DPS requires programs to offer 107 payment tokens before they can run. In principle 108 the tokens should be authenticated by a trusted 109 third party. At present these low level management 110 activities are carried out using a conventional hi- 111 erarchical approach. We hope to address adaptive 112 control of the o/s kernel supporting the DPS in 113 future work.

\section{Network level QoS}

Currently there is great interest in enabling the 116 Internet to handle low latency traffic more reliably 117 than at present. Many approaches, such as intserv 118 [7], rely on enabling the network to support some 119 type of connection orientation. This matches the 120 properties of older network applications, such as 121 telephony, well. However it imposes an unaccept- 122 able overhead on data applications that generate 123 short packet sequences. Given that traffic forecasts 124 indicate that by the end of the next decade tele- 125 phony will be $\approx 5 \%$ of total network traffic, and 126 short data sequences will be around $50 \%$ of net- 127 
128 work traffic, it does not seem likely that connec-

129 tion orientation will deliver optimal results.

130 A recent alternative has been to propose differ-

131 entiated services [8], an approach that is based on

132 using different forwarding rules for different classes

133 of packet, and maintaining the properties of the

134 best class by admission control at the ingress to the

135 network. There are difficulties however.

- Admission control does not work well with short packet sequences [9].

- The proposed algorithms assume Poisson burst intervals when real traffic is in fact fractional Gaussian $[10,11]$ and much harder to predict.

- The performance benefits can only be obtained if the distribution of demand is such that only a small proportion of the traffic wishes to use the better classes [12].

- The proposed classes typically propose a low loss, low latency class that uses a disproportionate proportion of the available network resources.

Despite the difficulties it is clear that differentiated services is currently the best available alternative. It therefore seems advisable to base any proposals for QoS management of active services on the diffserv approach. However, it also seems advisable to modify the approach and attempt to avoid some of the difficulties identified.

\section{4. Emergent approach to differentiated active ser- 157 vices}

158 We propose a new approach to differentiating 159 active services, controlled by an emergent control 160 algorithm. Users can request low latency at the 161 cost of high loss, moderate latency and loss, or 162 high latency and low loss by adjusting the time to 163 live (ttl) of the packets they send, either by ma164 nipulating the IP header or using a user defined 165 header extension. Short ttl packets will experience 166 high loss when the network is congested and long $167 \mathrm{ttl}$ packets will experience high delay when the 168 network is congested. Users cannot request low 169 loss and low delay together. This choice means 170 that all the classes of service we support have ap171 proximately the same resource cost, since the low 172 latency class does not rely on low utilization and we can set the utilization to be the same for all the 173 service classes. As a result we do not have to 174 consider complex admission control to ensure a 175 favourable demand distribution, and we do not 176 have to allocate significant resources to support a 177 minority service. Two adaptations are possible if 178 the performance is reduced by congestion; either 179 the application sends less packets or the applica- 180 tion persists until an application specific latency 181 cut-off is reached and then terminates the session. 182 Services such as telephony would use a low laten- 183 cy/high loss transport regime. This would require 184 the application to be more loss tolerant than at 185 present, however as mobile telephones demon- 186 strate this is not hard to achieve. Interoperation 187 with legacy telephones could be achieved by run- 188 ning loss tolerant algorithms (e.g., FEC) in the 189 PSTN/IP gateway. We do not believe that users 190 want an expensive low loss, low latency service. 191 The current PSTN exemplifies this service and 192 users are moving to VoIP as fast as they are able, 193 despite lower quality, in order to benefit from re- 194 duced prices.

Near optimal end to end performance across the network is obtained by enabling the servers to retain options in their application layer routing table for fast path, medium path and slow path (i.e., high loss medium loss and low loss). Packets are then quickly routed to a server whose performance matches their ttl. This avoids any need to perform flow control and force sequences of packets to follow the same route.

For this approach to work well the properties of the servers must adapt to local load conditions. Fast servers have short queues and high drop probabilities, slow servers have long queues and low drop probabilities. If most of the traffic is low latency the servers should all have short buffers and if most of the demand is low loss the servers should have long buffers. Adaptation of the buffer length can be achieved using an adaptive control mechanism [13], and penalising servers whenever a packet in their queue expires. Use of adaptive control has the additional advantage that it makes no assumptions about traffic distributions, and will work well in a situation where the traffic has significant long range dependency (LRD). This 
220 then resolves the final difficulty we noted with the

221 current network level diffserv proposals.

\section{5. Adaptive control}

223 Conventional control of dynamic systems is 224 based on monitoring state, deciding on the man225 agement actions required to optimise future state, 226 and enforcing the management actions. In classical 227 control the decision is based on a detailed knowl228 edge of how the current state will evolve, and a 229 detailed knowledge of what actions need to be 230 applied to move between any pair of states (the 231 equations of motion for the state space). Many 232 control schemes in the current Internet (SNMP, 233 OSPF) are based on this form of control. There is 234 also a less precise version known as stochastic 235 control, where the knowledge takes the form of 236 probability density functions, and statistical pre237 dictions. All existing forms of traffic management 238 are based on stochastic control, typically assuming 239 Poisson statistics.

240 Adaptive control [13] is based instead on 241 learning and adaptation. The idea is to compen242 sate for lack of knowledge by performing experi243 ments on the system and storing the results 244 (learning). Commonly the experimental strategy is 245 some form of iterative search, since this is known 246 to be an efficient exploration algorithm. Adapta247 tion is then based on selecting some actions that 248 the system has learnt are useful using some selec249 tion strategy (such as a Bayesian estimator) and 250 implementing the selected actions. Unlike in con251 ventional control, it is often not necessary to as252 sume the actions are reliably performed by all the 253 target entities. This style of control has been pro254 posed for a range of Internet applications includ255 ing routing [14], security [15,16], and fault 256 ticketing [17]. As far as we are aware the work 257 presented here is the first application of distributed 258 adaptive control to service configuration and 259 management.

260 Holland [18] has shown that genetic algorithms 261 (GAs) offer a robust approach to evolving effective 262 adaptive control solutions. More recently many 263 authors [19-21] have demonstrated the effective264 ness of distributed GAs using an unbounded gene pool and based on local action (as would be re- 265 quired in a multi-owner internetwork). However, 266 many authors, starting with Ackley and Littman 267 [22], have demonstrated that to obtain optimal 268 solutions in an environment where significant 269 changes are likely within a generation or two, the 270 slow learning in GAs based on mutation and in- 271 heritance needs to be supplemented by an addi- 272 tional rapid learning mechanism. Harvey [23] 273 pointed out that gene interchange (as observed in 274 bacteria [24,25]) could provide the rapid learning 275 required. This was recently demonstrated by 276 Furuhashi [26] for a bounded, globally optimised 277 GA. In previous work [27] we have demonstrated 278 that a novel unbounded, distributed GA with 279 "bacterial learning" is an effective adaptive control 280 algorithm for the distribution of services in an 281 active service provision system derived from the 282 ALAN. In this paper we demonstrate for the first 283 time that our adaptive control algorithm can de- 284 liver differentiated QoS in response to user sup- 285 plied metrics.

286

\section{Algorithm details}

Our proposed solution makes each DPS within the network responsible for its own behaviour. The active service network is modelled as a community of cellular automata. Each automaton is a single DPS that can run several programs (proxylets) requested by users. Each proxylet is considered to represent an instance of an active service. Each member of the DPS community is selfishly optimising its own (local) state, but this 'selfishness' has been proven as a stable model for living organisms [28]. Partitioning a system into selfishly adapting sub-systems has been shown to be a viable approach for the solving of complex and non-linear problems [29].

In this paper we discuss results from an implementation that supports up to 10 active services. The control parameters given below are examples provided to illustrate our approach. Our current implementation has 1000 vertices connected on a rectangular grid (representing the network of transport links between the DPSs). Each vertex can support a single server (i.e., host) supporting a
288

289

290

291

292

293

294

295

296

297

298

299

300

301

302

303

304

305

306

307

308

309 
310 single DPS, so the network can support up to 1000

311 DPS nodes. In reality a network node (router)

312 would be associated with many such hosts, possi-

313 bly organised as a cluster. In this work we are

314 assuming that the latency associated with a DPS is

315 significantly greater than that associated with bit

316 transport so we do not distinguish between DPS

317 links that are local and DPS links that are remote.

318 Each DPS has an amount of genetic material that

319 codes for the rule set by which it lives. There is a

320 set of rules that control the DPS behaviour. There

321 is also a selection of genes representing active

322 services. These define which services each node will

323 handle and can be regarded as pointers to the ac-

324 tual programs supplied by users. Each node can

325 hold up to eight services (the limit is similar to that

326 imposed by available RAM in commodity com-

327 puters, such as could be used in future server

328 clusters). The service genes also encode some

329 simple conditionals that must be satisfied for the

330 service to run. Currently each service gene takes

331 the form $\{x, y, z\}$ where:

342 The system is initialised by populating a random

343 selection of network vertices with DPSs (active 344 nodes), and giving each DPS a random selection of 345 the available service genes. Requests are then en346 tered onto the system by injecting a random se347 quence of characters (representing service 348 requests), at a mean rate that varies stochastically,

349 at each vertex in the array. If the vertex is popu-

350 lated by a DPS, the items join a queue. If there is

351 no DPS the requests are forwarded to a neigh-

352 bouring vertex. The precise algorithm for this

353 varies and is an active research area, however the

354 results shown here are based on randomly select-

355 ing a direction in the network and forwarding

356 along that direction till a DPS is located. This is

357 clearly sub-optimal but is easy to implement. The traffic arriving at each DPS using this model shows 358 some LRD, but significantly less than real WWW 359 traffic. Increasing the degree of LRD would be 360 straightforward. However, the necessary change 361 involves additional memory operations that slows 362 down the simulation and makes the results harder 363 to interpret. In any case inclusion of significant 364 LRD would not change the qualitative form of the 365 main results since the algorithm is not predictive 366 and makes no assumptions regarding the traffic 367 pdf. Each DPS evaluates the items that arrive in its 368 input queue on a FIFO principle. If the request at 369 the front of the queue matches an available service 370 gene, and the customer has included payment to- 371 kens equal to (or greater than) the cost for that 372 service in the DPS control rules, the service will 373 run. In the simulation the request is deleted and 374 deemed to have been served, and the node is re- 375 warded by a value equal to the specified cost of the 376 service. If there is no match the request is for- 377 warded and no reward is given. In this case the 378 forwarding is informed by a state table maintained 379 by the DPS using a node state algorithm. Packets 380 with a short $\mathrm{ttl}$ are forwarded to a DPS with a 381 short queue and packets with a long ttl are for- 382 warded to a DPS with a long queue. Each DPS is 383 assumed to have the same processing power, and 384 can handle the same request rate as all the others. 385 In the simulation time is divided into epochs (to 386 enable independent processing of several requests 387 at each DPS before forwarding rejected requests). 388 An epoch allows enough time for a DPS to execute 389 3-4 service requests, or decide to forward 30-40 390 (i.e., forwarding incurs a small time penalty). An 391 epoch contains 100 time units and is estimated to 392 represent $\mathrm{O}(100) \mathrm{ms}$. The busyness of each DPS is 393 calculated by combining the busyness at the pre- 394 vious epoch with the busyness for the current ep- 395 och in a $0.8-0.2$ ratio, and is related to the revenue 396 provided for processing a service request. For ex- 397 ample, if the node has processed three requests this 398 epoch ( 25 points each) it would have 75 points for 399 this epoch, if its previous cumulative busyness 400 value was 65 then the new cumulative busyness 401 value will be 67 . This method dampens any sudden 402 changes in behaviour. A brief schematic of this is 403 shown in Fig. 2. 
6

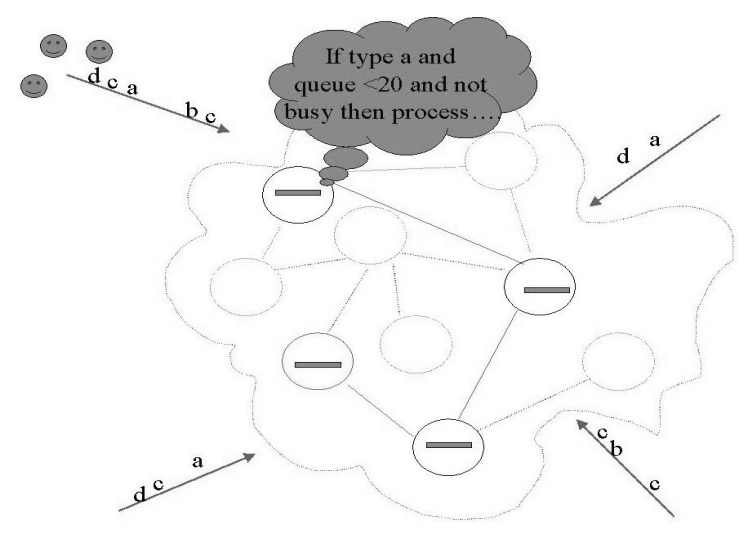

Fig. 2. Future network model.

405

406

407

408

409

410

411

412

413

414

415

416

performed every five epochs. Although the evaluation period is currently fixed there is no reason 438 why it should not also be an adaptive variable.

If the queue length or busyness is above a threshold (both 50 in this example), a random section of the genome is copied into a 'rule pool' accessible to all DPSs. If a DPS continues to exceed the threshold for several evaluation periods, it replicates its entire genome into an adjacent network vertex where a DPS is not present. Healthy bacteria with a plentiful food supply thus reproduce by binary fission. Offspring produced in this way are exact clones of their parent.

If the busyness is below a different threshold 441

The DPS also has rules for reproduction, evolution, death and plasmid migration. It is possible to envisage each DPS as a bacterium and each request for a service as food. The revenue earned when a request is handled is then analagous with the energy released when food is digested. This analogy is consistent with the metabolic diversity of bacteria, capable of using various energy sources as food and metabolising these in an aerobic or anaerobic manner.

Genetic diversity is created in at least two ways, mutation and plasmid migration. Mutation involves the random alteration of just one value in a single service gene, for example:" Accept request for service A if DPS $<80 \%$ busy" could mutate to "Accept request for service C if DPS $<80 \%$ busy" or alternatively could mutate to "Accept request for service A if DPS $<60 \%$ busy".

Plasmid migration involves genes from healthy individuals being shed or replicated into the environment and subsequently being absorbed into the genetic material of less healthy individuals. If plasmid migration does not help weak strains increase their fitness they eventually die. If a DPS acquires more than 4-6 service genes through interchange the newest genes are repressed (registered as dormant). This provides a long term memory for genes that have been successful, and enables the community to successfully adapt to cyclic variations in demand. Currently, values for queue length and cumulative busyness are used as the basis for interchange actions, and evaluation is
DPS is 'idle' for several evaluation periods, its active genes are deleted, if dormant genes exist, these are brought into the active domain, if there are no dormant genes the node is switched off. This is analogous to death by nutrient deprivation.

So if a node with the genome $\{a, 40,50 /$ $\mathrm{c}, 10,5\}$ has a busyness of $>50$ when analysed, it will put a random rule (e.g., c, 10,5) into the rule pool. If a node with the genome $\{\mathrm{b}, 2,30 / \mathrm{d}, 30,25\}$ is later deemed to be idle it may import that rule and become $\{\mathrm{b}, 2,30 / \mathrm{d}, 30,25 / \mathrm{c}, 10,5\}$.

\section{Experiments}

The basic traffic model outlined above was ad- 465 justed to enable a range of ttls to be specified. The 466 ttls used were 4, 7, 10, 15, 20, 25, 30, 40, 50, 100467 (expressed in epochs). Approximately the same 468 number of requests were injected at each $\mathrm{ttl}$. The 469 DPS nodes were also given an extra gene coding 470 for queue length, and penalised by four time units 471 whenever packets in the queue were found to have 472 timed out. A DPS with a short queue will handle 473 packets with a short ttl more efficiently since the ttl 474 will not be exceeded in the queue and the DPS will 475 not be penalised for dropping packets. Thus if 476 local demand is predominantly for short ttl DPS 477 nodes with short queues will replicate faster, and a 478 colony of short queue nodes will develop. The 479 converse is true if long $\mathrm{ttl}$ requests predominate. If 480 traffic is mixed a mixed community will develop. In 481 


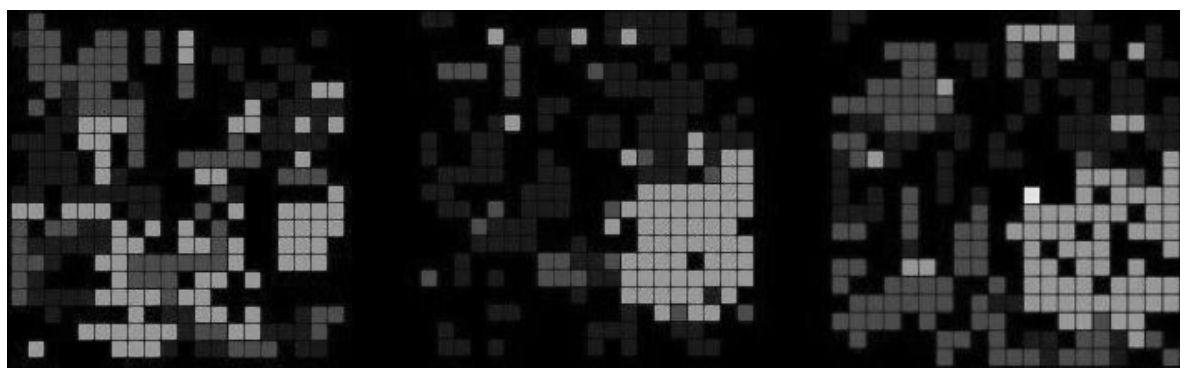

Fig. 3. Distribution of DPS nodes with short medium and long queues at three different times.

482 Fig. 3 the red dots represent DPS nodes with long 483 queues, the blue dots represent intermediate 484 queues and the green dots represent short queues. 485 It is clear that the distribution of capability 486 changes over time to reflect the distribution of 487 demand, in the manner described above.

488 In Fig. 4 we show the average request drop rate 489 across the network of bacteria illustrated in Fig. 3, 490 and compare the performance with a number of 491 alternative methods of distributing the active ser492 vices. The alternatives are:

- Random static placement of services at network nodes.

- Caching of requested services with a random replacement algorithm (Cache I).

- Caching of requested services using a least recently used replacement algorithm (Cache II).

499 The tests were performed at loads of $10 \%$ (low), $50040 \%$ (medium) and $80 \%$ (high). At low loads all the 501 algorithms offer similar performance levels. As 502 might be expected, at medium and high load our 503 algorithm is a significant improvement over ran-

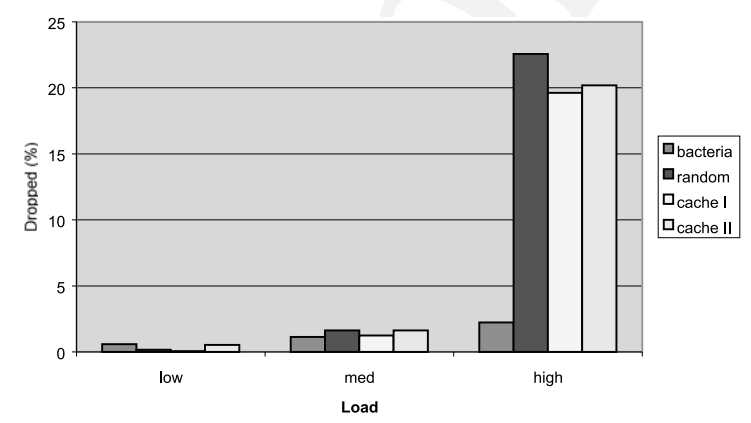

Fig. 4. Request drop rates for different distribution mechanisms. dom placement. More surprisingly it also signifi- 504 cantly outperforms caching. We believe this is due 505 to the small size of the caches. Each cache holds up 506 to eight services (i.e., the same as the bacteria). 507 This is intended to represent the number of 508 proxylets that can be held in the RAM of a low 509 spec PC, such as might be used in a commodity 510 based cluster at a network server farm. Since the 511 load time for proxylets is currently long $(\sim 1 \mathrm{~s})$ we 512 do not model disk based caching.

Fig. 5 shows the average end to end latency 514 experienced by service requests in our modelled 515 network, and compares it with the latency experi- 516 enced using the alternative active service distribu- 517 tion mechanisms listed above. As before the 518 adaptive bacterial approach is as good as the other 519 alternatives at low loads, and is clearly an im- 520 provement over the best alternative (standard 521 LRU based caching - CacheII) at medium and 522 high loads. We are therefore confident that our 523 algorithm is delivering a useful level of perfor- 524 mance.

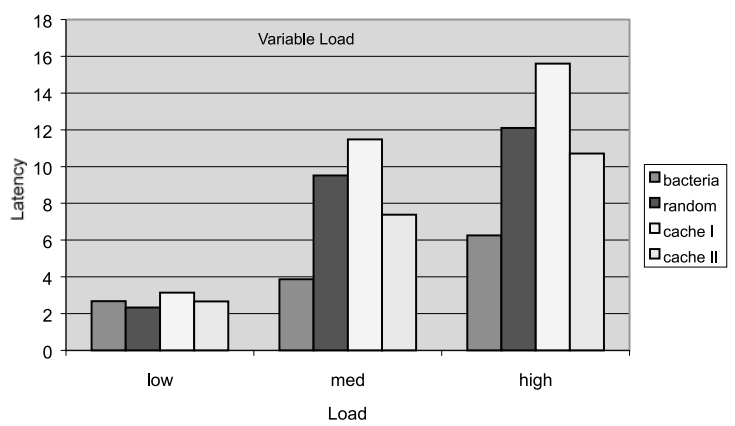

Fig. 5. Average latency of several approaches to distributing active services. 


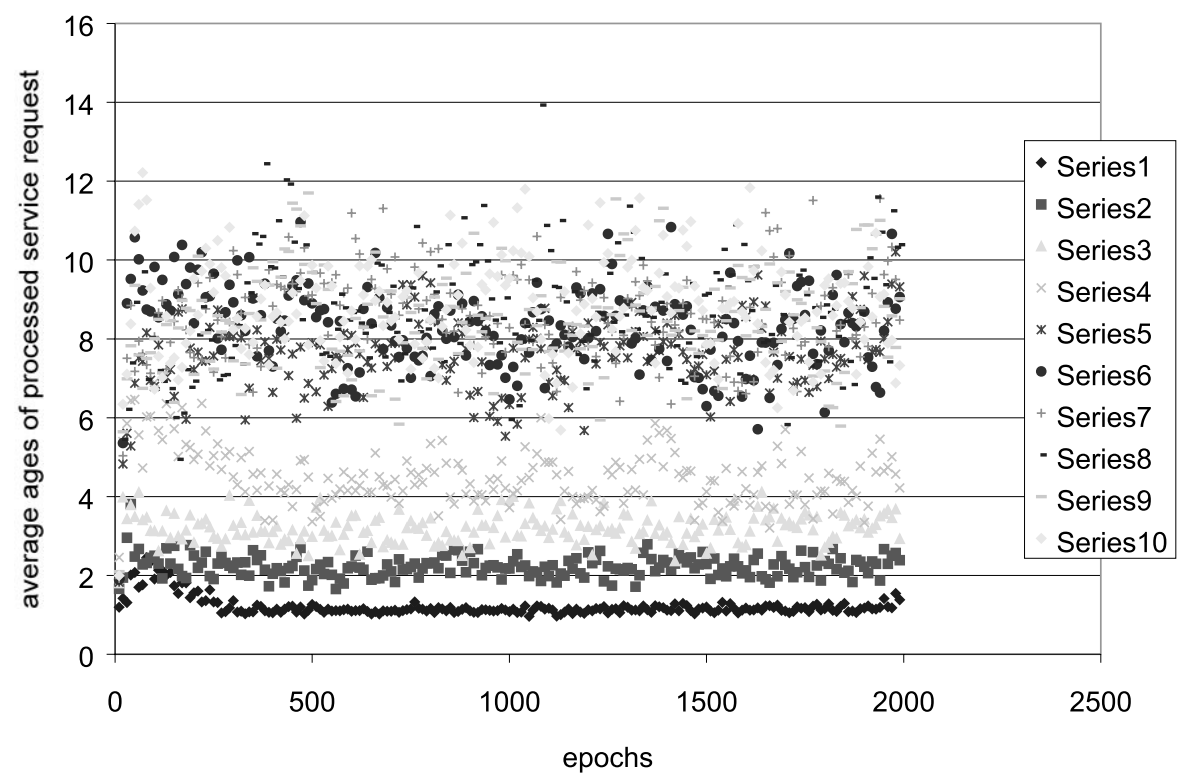

Fig. 6. Different latencies for requests with differing times to live.

526 Fig. 6 illustrates the differentiated QoS delivered

527 by the network of DPS nodes. The time taken to

528 process each request is shown on the $y$ access and

529 the elapsed system time is shown on the $x$ axis. It can be seen that the service requests with shorter 530 times to live are being handled faster than those 531 with a longer time to live, as expected. Fig. 7 shows 532 the expected corrollary. More service requests with 533

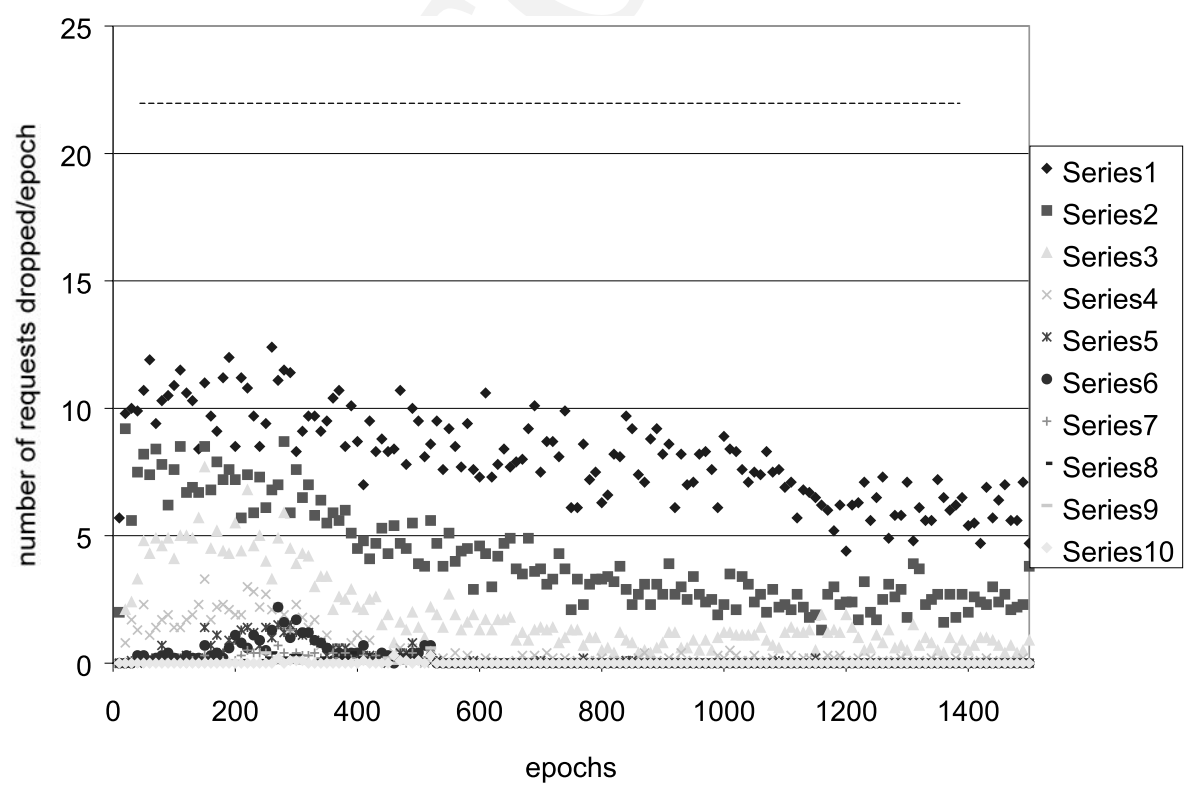

Fig. 7. Different dropping rates for requests with differing times to live. 


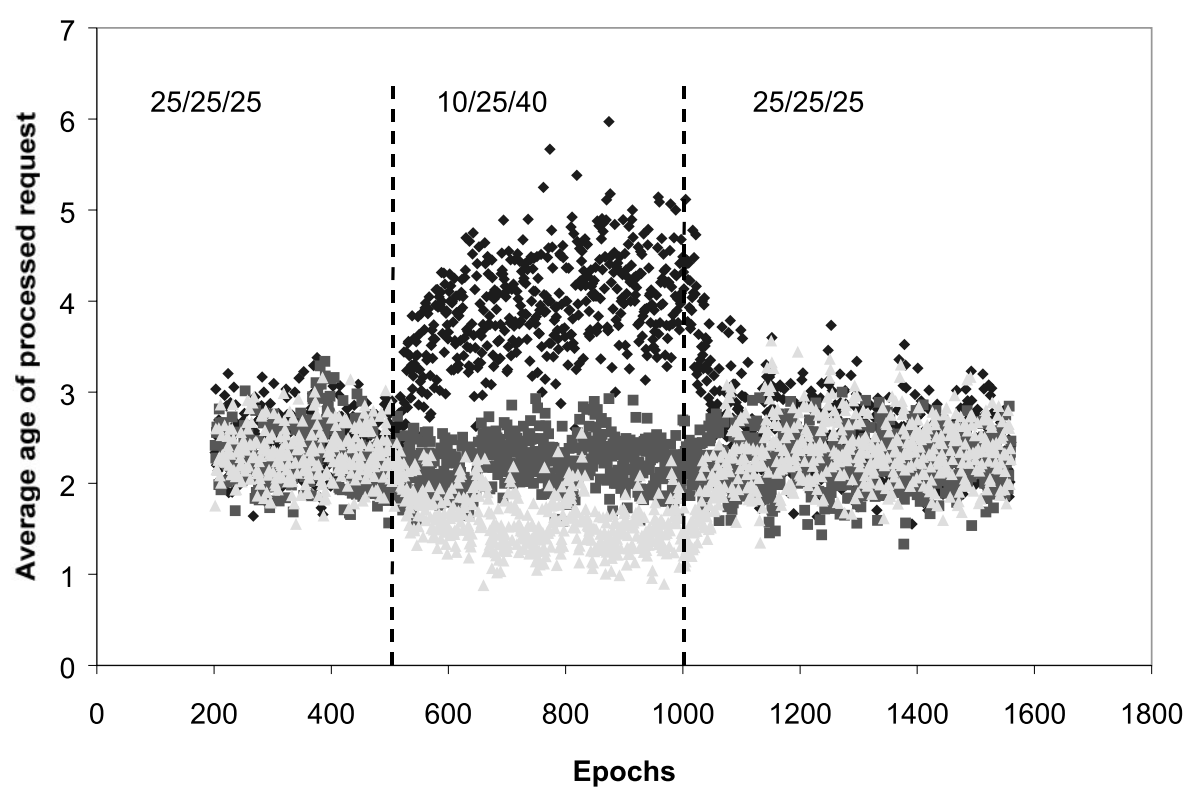

Fig. 8. Effects of different charging levels on age related QoS.

534 short ttls are being dropped. This is due to them 535 timing out, and is the essential down-side to 536 specifying a short ttl. Although the numbers of requests at each $\mathrm{ttl}$ value are roughly equal, fewer 537 short $\mathrm{ttl}$ requests are handled.

In addition to varying the latency and loss as- 539 sociated with service requests users may also wish 540

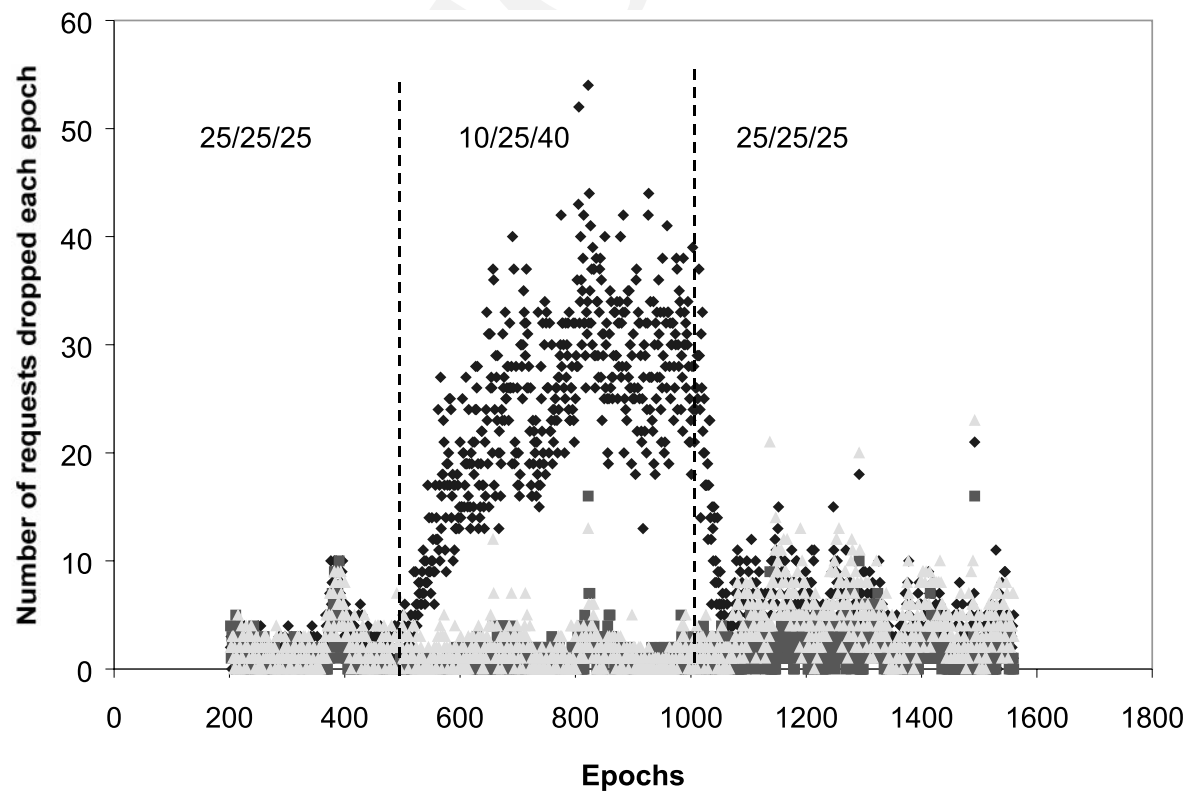

Fig. 9. Effects of different charging levels on dropping of requests. 
541 to vary the price they are willing to pay. In the

542 basic algorithm it was assumed that the network

543 provider allocated a reward to each DPS for pro-

544 cessing a service request. We investigated the im-

545 pact of allowing the DPS to collect a greater

546 reward. In the modified model the DPS is re-

547 warded by the amount of tokens the user includes

548 with the request. The traffic input was adjusted so

549 that requests for different services carried different

550 amounts of payment tokens. Initially the DPS

551 nodes were rewarded equally (25 'tokens') for each

552 of three services A, B and C. After 500 epochs the

553 rate of reward is changed so that each DPS is re-

554 warded four times as much for processing service

$555 \mathrm{C}$ (40 tokens) as it is for processing service A (10

556 tokens), with B staying at 25 . This is equivalent to

557 offering users a choice of three prices for a single

558 service. Fig. 8 shows the latency of service requests

559 for the three different service types.

560 It is apparent that within 100 epochs the average

561 latency for providing service $\mathrm{C}$ is reduced while the

562 latency for A is increased. Fig. 9 shows that re-

563 quests for service $A$ are also dropped (due to

564 timing out) more than requests for service B and

565 C. Before the change in reward the numbers of

566 DPSs handling each service were similar. After the

567 reward rate change the plasmids for handling

568 services $\mathrm{C}$ and $\mathrm{B}$ have spread much more widely

569 around the network at the expense of the plasmid

570 for the relatively unrewarding service A. After

5711000 epochs the rate of requests for all three ser-

572 vices was returned to the original state. It can be

573 seen, in both figures, that equality in quality of

574 service, both in terms of loss rate and latency,

575 quickly returned.

576 These last results indicate that the control 577 method could potentially be used for a range of 578 user specified parameters. We see no reason why 579 other parameters of interest could not be added to 580 the model, and are very encouraged by the initial 581 results. In particular we note that the latencies and 582 loss rates are comparable to those obtained in 583 many conventional approaches to differentiated 584 services, but many of the difficulties concerning 585 admission control have been avoided.

\section{Conclusions}

Our initial results show that the long-term self- 587 stabilising, adaptive nature of bacterial communi- 588 ties are well suited to the task of creating a stable 589 community of autonomous active service nodes 590 that can offer consistent end to end QoS across a 591 network. The methods used for adaptation and 592 evolution enable probabilistic guarantees for met- 593 rics such as loss rate and latency similar to what 594 can be achieved using more conventional ap- 595 proaches to differentiated services.

\section{References}

[1] M. Fry, A. Ghosh, Application layer active networking, Computer Networks 31 (7) (1999) 655-667.

[2] R. Giaffreda, N. Walker, R.Y. Ruiz, Performance of the 600 DNS name resolution infrastructure, in: Proceedings of the 601 IEE colloquium on Control of Next Generation Networks, 602 London, October 1999.

603

[3] I.W. Marshall, J. Hardwicke, H. Gharib, M. Fisher, P. 604 Mckee, Active management of multiservice networks, in: 605 Proceedings of the IEEE NOMS2000, pp. 981-983. 606

[4] P. Mckee, I.W. Marshall, Behavioural specification using 607 XML, in: Proceedings of the IEEE FTDCS '99, Capetown, 608 pp. $53-59$.

609

[5] I.W. Marshall, M. Fry, L. Velasco, A. Ghosh, Active 610 information networks and XML, in: S. Covaci (Ed.), 611 Active Networks, LNCS 1653, Springer, Berlin, 1999, pp. 612 $60-72$.

613

[6] D.G. Waddington, D. Hutchison, Resource partitioning in 614 general purpose operating systems, experimental results in 615 Windows NT, Operating Systems Review 33 (4) (1999) 52- 616 74.

617

[7] IETF Intserv charter: http://www.ietf.org/html.charters/ 618 intserv-charter.html.

619

[8] IETF Diffserv charter: http://www.ietf.org/html.charters/ 620 diffserv-charter.html.

[9] V. Jacobson, Support for differentiated services in router 622 designs, in: Proceedings of the Network Modelling in the 623 21st Century, Royal Society, December 1999.

[10] V. Paxson, S. Floyd, Wide area traffic: the failure of 625 poisson modelling, IEEE/ACM Transactions on Network- 626 ing 3 (3) (1995) 226-244.

627

[11] M. Crovella, A. Bestavros, Self-similarity in world wide 628 web traffic: evidence and possible causes, IEEE/ACM 629 Transactions on Networking 5 (6) (1997) 835-846. 630

[12] R. Gibbens, S.K. Sargood, F.P. Kelly, H. Azmoodeh, R. 631 MacFadyen, N. MacFadyen, An approach to service level 632 agreements for IP networks with differentiated services, 633 Philosophical Transactions of the Royal society A, 2000, 634 
submitted, and available at http://www.statslab.cam.ac.uk richard/research/papers/sla/.

[13] Y.Z. Tsypkin, Adaptation and learning in automatic systems, Mathematics in Science and Engineering, vol. 73, Academic press, New York, 1971.

[14] G. DiCaro, M. Dorigo, AntNet: distributed stigmergic control for communications networks, Journal of Artificial Intelligence Research 9 (1998) 317-365.

[15] D.A. Fisher, H.F. Lipson, Emergent algorithms - a new method of enhancing survivability in unbounded systems, in: Proceedings of the 32nd Hawaii International Conference on System Sciences, IEEE, 1999.

[16] M. Gregory, B. White, E.A. Fisch, U.W. Pooch, Cooperating security managers: a peer based intrusion detection system, IEEE Network 14 (4) (1996) 68-78.

[17] L. Lewis, A case based reasoning approach to the management of faults in telecommunications networks, in: Proceedings of the IEEE conference on Computer Communications, vol. 3, San Francisco, 1993, pp. 14221429.

[18] J.H. Holland, Adaptation in Natural and Artificial Systems, MIT press, Cambridge, MA, 1992.

[19] S. Forrest, T. Jones, Modeling complex adaptive systems with echo, in: R.J. Stonier, X.H. Yu (Eds.), Complex Systems Mechanisms of Adaptation, IOS Press, 1994, pp. 3-21.

[20] R. Burkhart, The swarm multi-agent simulation system, OOPSLA '94 Workshop on The Object Engine, September 7, 1994.

[21] J.U. Kreft, G. Booth, J.W.T. Wimpenny, BacSim, a simulator for individual-based modelling of bacterial colony growth, Microbiology 144 (1997) 3275-3287.

[22] D.H. Ackley, M.L. Littman, Interactions between learning and evolution, in: C.G. Langton, C. Taylor, J.D. Farmer, S. Rasmussen (Eds.), Artificial Life II, Addison-Wesley, Reading, MA, 1993, pp. 487-507.

[23] I. Harvey, The Microbial Genetic Algorithm, unpublished work, 1996, available at ftp://ftp.cogs.susx.ac.uk/pub/users/ inmanh/Microbe.ps.gz.

[24] S. Sonea, M. Panisset, A new bacteriology, Jones and Bartlett, 1983.

[25] D.E. Caldwell, G.M. Wolfaardt, D.R. Korber, J.R. Lawrence, Do bacterial communities transcend darwinism? Advances in Microbial Ecology 15 (1997) 105-191.
[26] N.E. Nawa, T. Furuhashi, T. Hashiyama, Y. Uchikawa, A 679 study on the relevant fuzzy rules using pseudo-bacterial 680 genetic algorithm, in: Proceedings of the IEEE Interna- 681 tional Conference on Evolutionary Computation, 1997. 682

[27] I.W. Marshall, C. Roadknight, Adaptive management of 683 an active services network, British Telecommunication 684 Technology Journal 18 (4) (2000) 78-84.

[28] R. Dawkins, The Selfish Gene, Oxford University Press, 686 Oxfoxd, 1976.

[29] S. Kauffman, The Origins of Order, Oxford University 688 Press, Oxford, 1993.

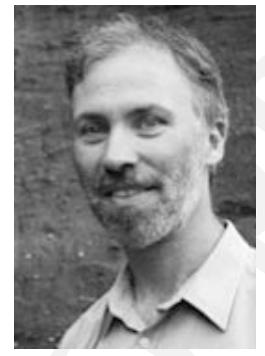

Ian Marshall is a senior research advisor at BT and visiting Professor of Telecommunications at South Bank University. Since 1997 he has been leading research on Ad-Hoc Systems, including active networks. This work currently includes the Alpine Research Initiative funded by BT, the fifth framework project Android and the Eurescom project Caspian, together with some smaller university research contracts. Previously he worked in optical networks, broadband networks, network strategy and distributed systems. He is a chartered engineer, a fellow of the Institute of Physics and the British Computer Society, a senior member of IEEE and a member of the ACM. He serves on several institute committees, on EPSRC and European research panels, and on numerous programme committees.

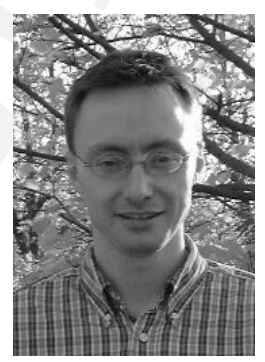

Chris Roadknight holds a B.Sc. in Biological Sciences and a M.Sc. in Computer Sciences from Manchester University and was recently awarded a Doctorate for his thesis on 'Transparent Neural Network Data Modelling' by Nottingham Trent University. He joined BT in 1997 and initially worked on characterising WWW requests and user behaviour, with applications to proxy cache performance modelling. More recently his work within the programmable networks lab has fofor active network management.

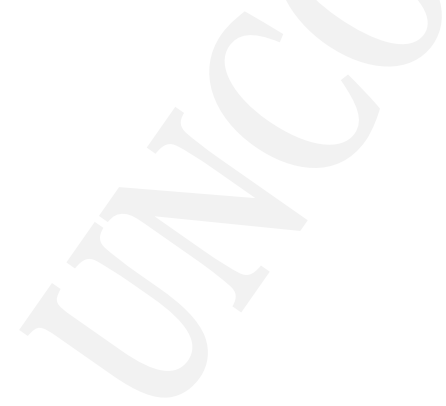

